A list of Working Papers on the last pages

No. 231, 1989

LOSS OF STABILITY AND EMERGENCE OF CHAOS IN DYNAMICAL SYSTEMS

by

Russell A. Johnson

Paper prepared for The Second International Workshop on Dynamic Sciences, June 5-16, 1989, at IUI, Stockholm

November, 1989 


\title{
LOSS OF STABILITY AND EMERGENCE OF CHAOS IN DYNAMICAL SYSTEMS*
}

\author{
RUSSELL A. JOHNSON†
}

I. Introduction. The purpose of these notes is to give a brief overview of some of the basic ideas involved in studying the breakdown of stability in finite-dimensional dynamical systems. We will at the end arrive at one frontier of modern research: the problem of determining how "chaotic" invariant sets can come into existence in such dynamical systems.

Let us first recall that a dynamical system consists of a set ("phase space") $\mathrm{X}$, an abelian group $(T,+)$, and a mapping $\tau: X \times T \rightarrow X$ which satisfies the following properties: (i) $\tau(x, 0)=x$ for all $x \in X$; (ii) $\tau_{t} \circ \tau_{s}=\tau_{t+s}$ for all $t, s \in T$, where $\tau_{t}(x) \stackrel{\text { def }}{=} \tau(x, t)$. The second property is called the group property. If $X$ and $T$ are topological spaces, then $\tau$ is required to be continuous in both variables. In this case, $t \rightarrow \tau_{t}$ defines a one parameter group of homeomorphisms of $X$.

We will only consider dynamical systems in which $X$ is a subset of $\mathbf{R}^{\boldsymbol{k}}$ and $T$ is either the real line $\mathbf{R}$ on the integers $\mathbf{Z}$ (with addition as the group operation). If $T=\mathbf{Z}$, we call the dynamical system discrete. Observe that, if $T=\mathbf{Z}$, and if $f: X \rightarrow X$ is defined by $f(x)=\tau(x, 1)$, then $f^{n}(x)=\varphi(x, n)$ for all $n \in \mathbf{Z}, x \in X$. Thus a discrete dyramical system is completely determined by a single homeomorphism $f$ of $X$. We can and will express a discrete dynamical systern in terms of a difference equation:

$$
x_{n+1}=f\left(x_{n}\right) \quad(-\infty<n<\infty)
$$

where $x_{n}=f^{n}\left(x_{0}\right) \equiv \tau\left(x_{0}, n\right)$.

If $T=\mathbf{R}$, we call the dynamical system continuous. A large class of continuous dynamical systems is obtained by solving differential equations of the form

$$
\begin{aligned}
& x^{\prime}=f(x) \quad x \in \mathbf{R}^{k} \\
& x(0)=x_{0}
\end{aligned}
$$

Let $\tau\left(x_{0}, t\right)$ denote the solution of $(1)$ with initial condition $x_{0}$. Assuming that solutions of (1) exist for all time $-\infty<t<\infty$ and are unique for all initial conditions $x_{0} \in \mathrm{R}^{k}$, it is easily seen that $\left(x_{0}, t\right) \rightarrow \tau\left(x_{0}, t\right)$ defines a dynamical system with phase space $R^{k}$ and group $\mathbf{R}$.

\footnotetext{
*This work was completed and typed while the author was visiting the Institute for Mathematics and its Applications at the University of Minnesota.

†Institute for Mathematics and its Applications, Minneapolis, Minnesota 55455 and Cniversity of Southern California, Los Angeles, CA 90089
} 
Having defined what we mean by a dynamical system, we next consider the concept of stability. Consider a one parameter family of differential resp. difference equations

$$
\begin{gathered}
x^{\prime}=f_{\mu}(x) \\
x_{n+1}=f_{\mu}\left(x_{n}\right)
\end{gathered}
$$

Let $Y=Y_{\mu}$ be a family of compact invariant sets (invariance means that, if $x_{0} \in Y_{\mu}$, then so is $\tau_{\mu}\left(x_{0}, t\right)$ for all $t \in \mathbf{R}$ resp. for all $t \in \mathbf{Z}$ ). Suppose that the $Y_{\mu}$ are asymptotically stable for $\mu<0$. This means that, for each $\mu<0$, there is an open set $V=V_{\mu} \supset Y_{\mu}$ such that, if $x_{0} \in V$ and if $t_{n} \rightarrow \infty$ is a sequence for which $\tau_{\mu}\left(x_{0}, t_{n}\right)$ converges, then $\lim _{n \rightarrow \infty} \tau_{\mu}\left(x_{0}, t_{n}\right) \in Y_{\mu}$. Suppose on the other hand that $Y_{\mu}$ is not asymptotically stable for $\mu>0$. In this situation we say that stability is lost (at $\mu=0$ ). We pose the basic question: is there an asymptotically stable invariant set for $\mu>0$ ? If so, what are its properties?

Bifurcation theory addresses this general question. As we will see, if each $Y_{\mu}$ corresponds to a fixed point or a periodic solution of the differential resp. difference equation, then our question can be answered in a satisfactory way. For more general invariant sets $Y_{\mu}$, however, much less information is available. It does appear that hyperbolic invariant sets appear quite frequently when a general family of invariant sets $Y_{\mu}$ loses stability.

We can now explain the plan of these notes. First, we describe some basic elements of the theory of bifurcation, in particular fixed-point bifurcation and Hopf bifurcation. Second, we discuss hyperbolic invariant sets and their properties; they exhibit highly chaotic behavior. Finally, we indicate how loss of stability can lead to the occurrence of hyperbolic invariant sets.

II. Bifurcation Theory. We return to the equations (2), and once again let $Y_{\mu}$ be a family of compact invariant sets. It is natural to begin with the simplest imaginable case, namely each $Y_{\mu}$ is a single point. Without loss of generality we can assume that $Y_{\mu}=\{0\}$, the origin in $\mathbf{R}^{k}$. Thus $f_{\mu}(0)=0$.

We first consider continuous dynamical systems. It is convenient to consider two (closely related) examples.

Example 1. $x^{\prime}=f_{\mu}(x)=-x^{3}+\mu x \quad(\mu \in \mathbf{R}, x \in \mathbf{R})$.

This is a one-dimensional example: $k=1$. We have $f_{\mu}(0)=0$. There is a principle of linearized stability which says that the stability of $x=0$ is determined by the sign of $f_{\mu}^{\prime}(0)$. In fact, since $f_{\mu}^{\prime}(0)<0$ if $\mu<0$ and $f_{\mu}^{\prime}(0)>0$ if $\mu>0$, we can conclude that $x=0$ is asymptotically stable for $\mu<0$ and unstable for $\mu>0$.

We ask: is there a stable invariant set if $\mu>0$ ? It is natural to look for stable fixed points. Set $0=-x^{3}+\mu x=x\left(-x^{2}+\mu\right)$. The solutions are $x=0$ and $x= \pm \sqrt{\mu}$, the latter being real exactly when $\mu>0$. By the principle of linearized stability, $x= \pm \sqrt{\mu}$ are stable for $\mu>0$. Thus we see that, at $\mu=0, x=0$ "bifurcates" to not one, but two stable fixed 
points. Stability is "transferred" from $x=0$ to the fixed points $x= \pm \sqrt{\mu}$. One calls the bifurcation supercritical and illustrates it with the diagram in Figure 1. This diagram is called a pitchfork for obvious reasons.

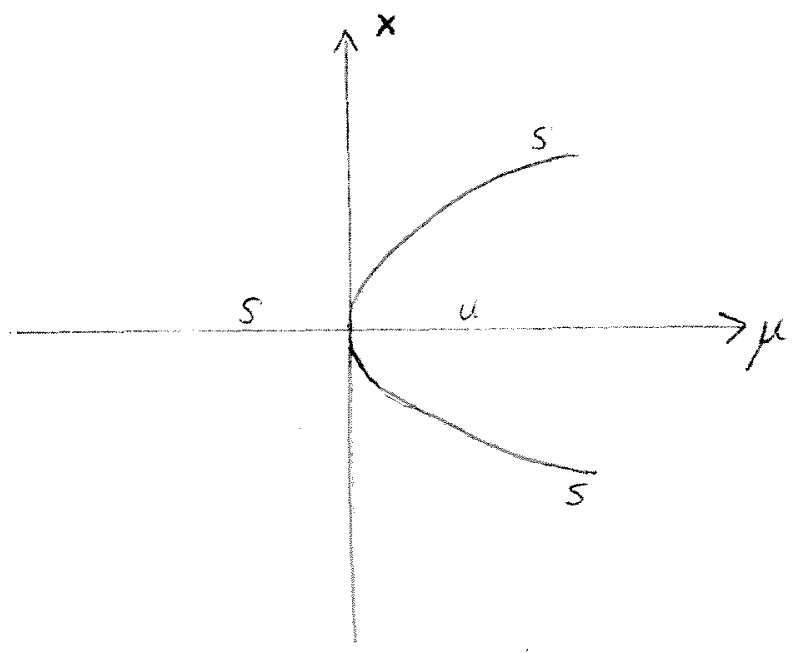

Figure 1

Example 2. $x^{\prime}=x^{3}+\mu x \quad(x, \mu \in \mathbf{R})$.

The only difference between examples 1 and 2 is the sign before $x^{3}$. Once again $x=0$ is stable for $\mu<0$ and unstable for $\mu>0$. However, this time it is easily seen (by direct integration of the differential equation) that, for $\mu>0$, every non-zero solution exists only on a finite time interval! Thus there are no invariant sets at all (other than $x=0$ ), bence there is no stable stable invariant set if $\mu>0$.

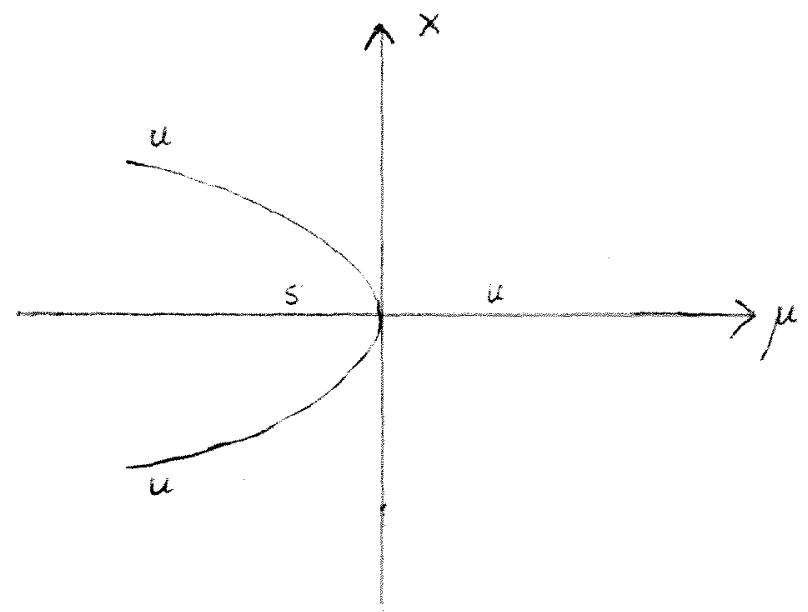

Figure 2

Note that $0=x^{3}+\mu x$ has the non-trivial solutions $x= \pm \sqrt{-\mu}$ if $\mu<0$. By the principle of linearized stability, these solutions are unstable. One calls this a subcritical bifurcation and illustrates it with the diagram of Figure 2. 
Examples 1 and 2 may seem rather special, but in fact the diagrams of Figures 1 and 2 occur quite frequently in applied problems. Indeed, there is a large class of onedimensional vector fields for which the "generic" bifurcation pattern of a one-parameter family is reflected by these diagrams. This class is that of smooth vector fields $f: \mathbf{R} \rightarrow \mathbf{R}$ which are antisymmetric with respect to $x=0$ : thus $f(-x)=-f(x)$. On speaks of " $Z_{2}$ symmetry" in this case. We refer to the excellent book of Golubitsky and Schaefer [4] for a detailed discussion, and content ourselves with the following motivational arguments.

Suppose that $f_{\mu}(x)=P_{\mu}(x)$ where each $P_{\mu}(x)$ is a real polynomial of degree $\geq 3$ such that $P_{\mu}(-x)=-P_{\mu}(x)$. Suppose further that, if $\mu<0$, then $P_{\mu}(x)$ has exactly one real root, which we take to be $x=0$. If $P_{\mu}(x)$ has more than one real root for $\mu>0$, then it will have (generically) exactly three real roots for $\mu>0$, since new roots arise from the coalescence of complex conjugate roots at $\mu=0$. Now, suppose $x=0$ is stable for $\mu<0$ but unstable for $\mu>0$. Then a simple argument using the principle of linearized stability shows that generically, a supercritical pitchfork bifurcation will occur at $\mu=0$. (Write $P_{\mu}(x)=x Q_{\mu}(x)$ where $Q_{\mu}(x)$ is an even polynomial, and investigate $d P_{\mu} / d x$.)

Suppose on the other hand that $P_{\mu}(x)=0$ for all $\mu$, that $x=0$ is the only real root for $\mu>0$, and that $x=0$ is stable for $\mu<0$. Then, generically, one finds a subcritical pitchfork bifurcation at $\mu=0$.

We finish our discussion of fixed-point bifurcation for continuous systems by noting that a large number of higher-dimensional problems can be reduced to a one-dimensional problem. Briefly, let $f_{\mu}: \mathbf{R}^{k} \rightarrow \mathbf{R}^{k}$ be a one-parameter family of vector fields with $f_{\mu}(0)=$ 0 , and let $A_{\mu}=D f_{\mu}(0)$ where $D$ stands for the usual Fréchet derivative. Suppose that the eigenvalues of $A_{\mu}$ have negative real parts if $\mu<0$ (this guarantees that $x=0$ is asymptotically stable if $\mu<0$ ). Suppose further that exactly one eigenvalue $\lambda(\mu)$ (necessarily real) passes through $\lambda=0$ at $\mu=0$. Then the Lyapunov-Schmidt procedure [8] permits one to reduce the $k$-dimensional bifurcation problem to one dimension, essentially by projecting onto the one-dimensional eigenspace $E \subset \mathbf{R}^{k}$ of $A_{0}$ which corresponds to $\lambda(0)=0$.

Next we turn our attention to fixed-point bifurcation for a one-parameter family of maps $f_{\mu}: \mathbf{R} \rightarrow \mathbf{R}$. Once again there is a principle of linearized stability: if $g: \mathbf{R} \rightarrow \mathbf{R}$ is smooth, and if $g\left(x_{0}\right)=x_{0}$, then $x_{0}$ is asymptotically stable if $\left|g^{\prime}\left(x_{0}\right)\right|<1$ and is unstable if $\left|g^{\prime}\left(x_{0}\right)\right|>1$.

Assume now that $f_{\mu}\left(x_{\mu}\right)=x_{\mu}$ for $\mu \in \mathbf{R}$, and that $\left|f^{\prime}\left(x_{\mu}\right)\right|<1$ for $\mu<0$ and $\left|f^{\prime}\left(x_{\mu}\right)\right|>1$ for $\mu>0$. At $\mu=0$ one must have $f_{0}^{\prime}\left(x_{0}\right)= \pm 1$. It can be shown that, if $f_{0}^{\prime}\left(x_{0}\right)=+1$, then there is a theory of bifurcation analogous to that already discussed for continuous systems. In particular, there is a certain ubiquity in the appearance of supercritical and subcritical bifurcation diagrams.

If $f_{0}^{\prime}\left(x_{0}\right)=-1$, however, there arises a new phenomenon, that of period doubling. Indeed, since $f_{\mu}(x)=f_{\mu}^{\prime}\left(x_{\mu}\right)\left(x-x_{\mu}\right)+\ldots$, and since $f_{\mu}^{\prime}\left(x_{\mu}\right) \simeq-1$ for $|\mu|$ small, we see that the orbit $\left\{f_{\mu}^{n}(x)\right\}$ will jump from one side to the other of $x_{\mu}$ if $x$ is near $x_{\mu}$. Moreover, 
if appropriate conditions are placed on the non-linear terms in $f_{\mu}(x)$, one can ensure that $\left\{f_{\mu}^{n}(x)\right\}$ cannot move too far from $x_{\mu}$, if $x-x_{\mu}$ is small. This indicates a supercritical bifurcation to a stable periodic orbit of period 2 .

Rather than pursuing further these general considerations, let us review a well-known special case when supercritical period doubling occurs. Namely consider the one-parameter family of interval maps

$$
f_{\mu}:[-1,1] \rightarrow[-1,1]: f_{\mu}(x)=1-\mu x^{2}(0 \leq \mu \leq 2)
$$

For each $\mu \in[0,3 / 4], f_{\mu}$ has a fixed point $x_{\mu} \in[-1,1]$ which is easily seen to be stable. Write $\mu_{0}=3 / 4$; one can see that $x_{\mu_{0}}=2 / 3$ and $f_{\mu_{0}}^{\prime}\left(x_{\mu_{0}}\right)=-1,\left|f_{\mu}^{\prime}\left(x_{\mu}\right)\right|>1$ for $\mu>\mu_{0}$. It be can shown that, for small $\mu-\mu_{0}>0$, there is a stable period point of period 2 for $f_{\mu}$. (We probably should remark at this point that the $f_{\mu}$ are not homeomorphisms of $[-1$, 1], but so what?)

It is well-known what happens when $\mu$ increases from $\mu_{0}=3 / 4$ : there is a sequence $\mu_{0}<\mu_{1}<\cdots<\mu_{n}<\ldots$ of parameter values at which a periodic point of period $2^{n}$ loses stability and a periodic point of period $2^{n+1}$ gains stability. That is, there is an infinite sequence of period doublings. It is known that $\mu_{n} \rightarrow \mu_{\infty} \simeq 1.40155$. In fact, one has the following table which we copy from the excellent book of Collet-Eckman [2]:

$\begin{array}{cccc}n & \mu_{n} & \mu_{n}-\mu_{n-1} & \frac{\mu_{n}-\mu_{n-1}}{\mu_{n+1}-\mu_{n}} \\ 0 & .75 & - & - \\ 1 & 1.25 & .5 & 4.23373 \\ 2 & 1.36807 & .11809 & 4.55150 \\ 3 & 1.39404 & .02594 & 4.64581 \\ 4 & 1.39963 & .00558 & 4.66393 \\ 5 & 1.40082 & .00119 & 4.66810 \\ 6 & 1.40108 & .00025 & 4.66896 \\ & \vdots & & \vdots \\ & \rightarrow \mu_{\infty} \simeq 1.40155 & & \rightarrow \delta=4.66920\end{array}$

The number $\delta$ is called Feigenbaum's constant. Reinterpreting the convergence of the ratios $\left(\mu_{n}-\mu_{n-1}\right) /\left(\mu_{n+1}-\mu_{n}\right)$ one has:

$$
\left|\mu_{n}-\mu_{\infty}\right| \sim \operatorname{const} \cdot \delta^{-n}
$$

Thus $\mu_{n}$ converges to $\mu_{\infty}$ at an exponential rate determined by $\delta$.

Now, it is a remarkable fact that exactly this same number $\delta$ occurs in many other one-parameter families of maps $\left\{f_{\mu}\right\}$ of the interval, in exactly the same role: $\left|\mu_{n}-\mu_{\infty}\right| \sim$ const $\cdot \delta^{-n}$. What is the explanation of this phenomenon? 
Following Feigenbaum [3] as discussed by Collet-Eckmann [2], we introduce the space $P$ of symmetric unimodal functions $f:[-1,1] \rightarrow[-1,1]$. This means that $f$ is symmetric (i.e., $f(-x)=f(x)$ ) and satisfies the following conditions: (i) $f$ is a $C^{3}$ function; (ii) $f(0)=1$; (iii) $f$ is increasing on $[-1,0)$ and decreasing on $(0,1]$; (iv) $f^{\prime}(x) \neq 0$ if $x \neq 0$.

Consider the subspace $V$ of $\mathbf{P}$ which consists of functions $f$ such that, if $a=-f(1)$ and $b=f(a)$, then $0<a<b<1$ and $f(b)=f(f(a))<a$. The content of these rather unusual-looking conditions is the following: (v) $f \circ f=f^{2}$ maps $[-a, a]$ into itself; (vi) $x \rightarrow(-1 / a) f \circ f(a x)$ is unimodal and symmetric. One may experiment with the function $f(x)=1-1.2 x^{2}$, which satisfies the stated conditions.

$\mathrm{By}(\mathrm{v})$ and (vi), it makes sense to define the renormalization transformation

$$
\mathcal{F}: V \rightarrow \mathbf{P}:(\mathcal{F} f)(x)=(-1 / a) f \circ f(a x)
$$

The renormalization transformation is of use in studying maps $f \in V$ which are superstable, i.e. for which $x=0$ is a periodic point. (Since $f^{\prime}(0)=0, x=0$ is necessarily a stable periodic point if it is periodic.) In fact, one can show that, if $f \in V$, then $f$ is superstable of minimal period $p$ if and only if $\mathcal{F f}$ is superstable of minimal period $p / 2$.

Now we indicate the strategy outlined by Feigenbaum and carried out by ColletEckermann, arrived at explaining the universality of $\delta$. The first step is to show that $\mathcal{F}$ admits a fixed point $\phi \in V$. The second is to show that the fixed point is hyperbolic: in fact, the derivative $D_{\phi} \mathcal{F}$ has one simple eigenvalue which is greater than one (and this eigenvalue is exactly $\delta$ ), while the rest of the spectrum of $D_{\phi} \mathcal{F}$ lies strictly inside the unit disc. This implies that there is a one-dimensional unstable manifold $W^{\boldsymbol{u}}$ passing through $\phi$, and a codimension 1 stable manifold $W^{s}$ passing through $\phi$ (Fig. 3). The third step is to consider the submanifold $\Sigma_{1}=\{f \in V \mid f(1)=0\}$, i.e. the set of $f \in V$ which are superstable of period 2. The inverse images $\Sigma_{j}=\mathcal{F}^{-(j-1)} \Sigma_{1}$ consist of functions $f$ which are superstable of period $2^{j}$. One can show that $\Sigma_{1}$ intersects $W^{u}$ transversally, as indicated in Fig. 3. Since $\Sigma_{j}=\mathcal{F}^{-(j-1)} \Sigma_{1}$, the manifolds $\Sigma_{j}$ must tend to the stable manifold $W^{s}$ as $j \rightarrow \infty$. 


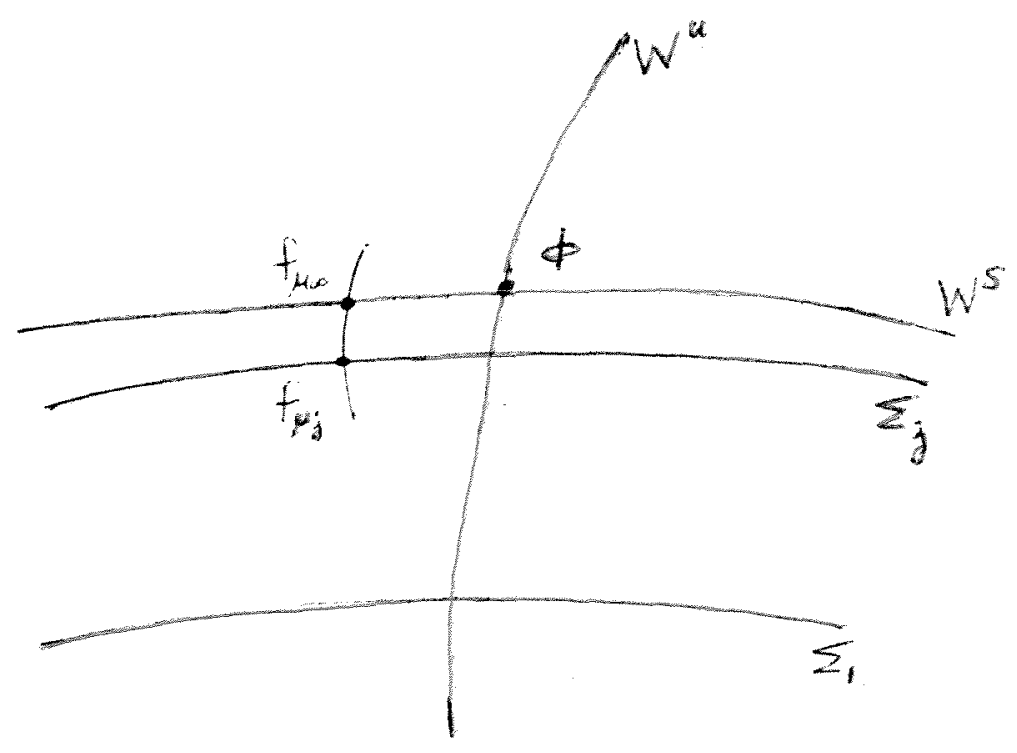

FIGURE 3

Finally, consider a curve $\mu \rightarrow f_{\mu}$ in $\mathbf{P}$ which intersects $W^{s}$ transversally. Let $\mu_{\infty}$ be the value at which $f_{\mu_{\infty}} \in W^{s}$, and let $\mu_{j}$ be the value at which $f_{\mu_{j}} \in \Sigma_{j}$. If $j$ is large, then $\left|\mu_{j}-\mu_{\infty}\right| \sim$ const.$\delta^{-j}$.

To formulate this picture precisely and prove that it is correct is is a non-trivial matter and the third chapter of [2] is devoted to the project. Nevertheless the sketch we have given should give an idea of the origin of what at first seems so mysterious: the universality of $\delta$.

Until now we have considered one-dimensional bifurcation problems, or (remembering our remark about the Liapounov-Schmidt procedure) problems in which "stability is lost in one direction". We now turn to problems in which stability is lost in two directions. In this situation, a frequently-encountered phenomenon is the so-called Hopf bifurcation.

We begin once more with an example. Let

$$
\vec{x}^{\prime}=\left(\begin{array}{c}
y \\
\left(\mu-x^{2}\right) y-x
\end{array}\right)=f_{\mu}(\vec{x}) .
$$

Note that $f_{\mu}$ fixes the origin for all $\mu: f_{\mu}(\overrightarrow{0})=\overline{0}$. The stability of the origin is determined by the eigenvalues of the derivative:

$$
D f_{\mu}(\overrightarrow{0})=\left(\begin{array}{cc}
0 & 1 \\
-1 & \mu
\end{array}\right) .
$$


The eigenvalues are $\lambda=1 / 2\left(\mu \pm \sqrt{\mu^{2}-4}\right)$, so $\operatorname{Re} \lambda(\mu)=\mu / 2$. Thus $\vec{x}=\overrightarrow{0}$ is stable if $\mu<0$ and unstable if $\mu>0$. Once again we pose the question: is there a stable invariant set for $\mu>0$ ?

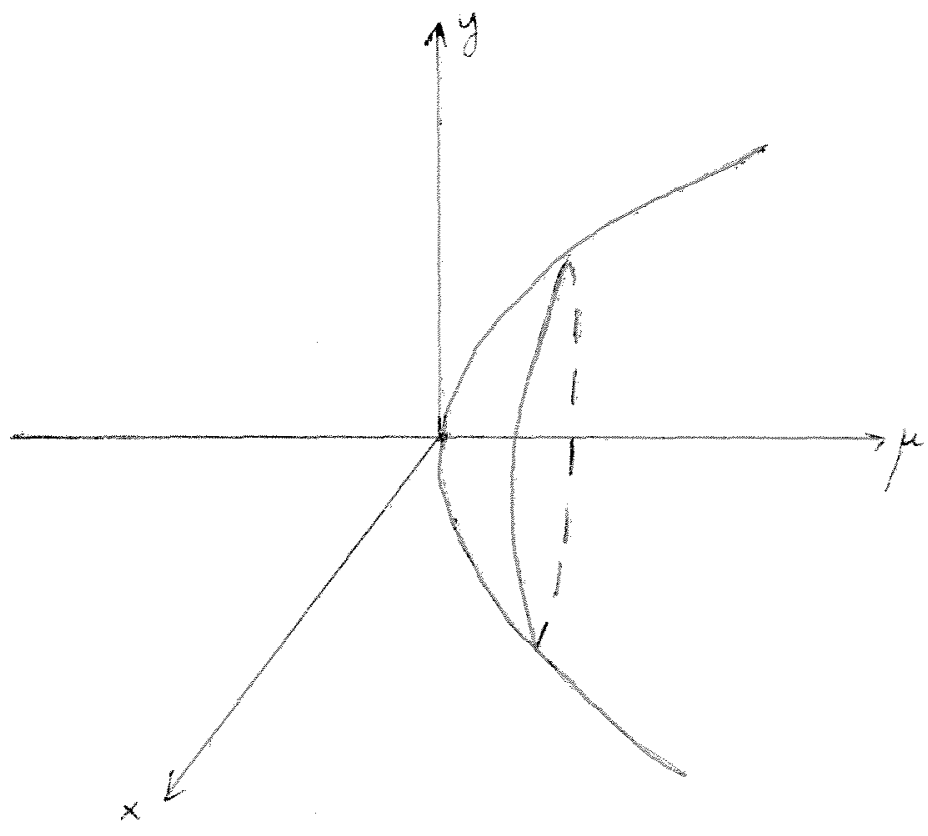

FIGURE 4

The answer is yes; in fact there is a stable invariant curve, diffeomorphic to a circle, with radius $r=|\vec{x}| \simeq \sqrt{\mu}$. One can see this by a slightly nontrivial analysis (which we omit) of the radial equation

$$
r^{\prime}=r \sin ^{2} \theta\left(\mu-r^{2} \cos ^{2} \theta\right)
$$

We have here an example of a supercritical Hopf bifurcation (Fig. 4). The invariant curve is defined by a periodic solution.

It is also easy to construct examples exhibiting a subcritical Hopf bifurcation; thus the invariant curve exists for $\mu<0$ and is unstable.

Consider now a general one-parameter family of differential equations

$$
x^{\prime}=f_{\mu}(x) \quad\left(x \in \mathbf{R}^{k}, \mu \in \mathbf{R}\right)
$$

Suppose that $f_{\mu}(0)=0$ for all $\mu$, and that the real parts of the eigenvalues of $D f_{\mu}(\vec{x})$ are all negative for $\mu<0$. Thus the origin is asymptotically stable for $\mu<0$. Suppose further that two complex conjugate eigenvalues $\lambda(\mu), \overline{\lambda(\mu)}$ cross the imaginary axis with non-zero 
speed at $\mu=0$, while all the other eigenvalues are bounded away from the imaginary axis. Precisely, suppose that

$$
\operatorname{Re} \lambda(0)=0, \operatorname{Im} \lambda(0) \neq 0, d / d \mu \operatorname{Re} \lambda(\mu)>0,
$$

while $\operatorname{Re} \tilde{\lambda}(0)<0$ for all other eigenvalues $\tilde{\lambda}(\mu)$.

The theorem of Hopf-Andronov states that, if the above conditions are satisfied, and if the origin is a "weak attractor" at $\mu=0$, then a supercritical Hopf bifurcation occurs: for small $\mu>0$ there is a stable invariant curve for $x^{\prime}=f_{\mu}(x)$ (which is in fact a periodic solution). Similarly, if the origin is a "weak repeller", then there is a subcritical Hopf bifurcation. We refer to the book of Marsden-McCracken [8] for details.

We now consider the somewhat more detailed theory of Hopf bifurcation for maps, due to R. Sacker [11]. We follow the treatment in the book of G. Iooss [6]. For convenience we consider only planar maps: $k=2$.

Consider then a one-parameter family of mappings

$$
f_{\mu}: \mathbf{R}^{2} \rightarrow \mathbf{R}^{2} \quad(\mu \in \mathbf{R})
$$

Once again write $\vec{x}=\left(\begin{array}{l}x \\ y\end{array}\right)$ for the position vector. Assume that $f_{\mu}(\overrightarrow{0})=\overrightarrow{0}$ for all $\mu$.

Suppose now that $\left\{f_{\mu}\right\}$ satisfies the following hypotheses.

(H1) $D f_{0}(\overrightarrow{0})$ has complex conjugate eigenvalues $\lambda_{0}, \bar{\lambda}_{0}$ with $\left|\lambda_{0}\right|=1$ and $\lambda_{0} \neq \pm 1$.

(H2) $d / d \mu|\lambda(\mu)|>0$ at $\mu=0$.

In particular $\vec{x}=\overrightarrow{0}$ is stable for $\mu<0$ and unstable for $\mu>0$.

Let us identify the complex plane $C$ with $\mathbf{R}^{2}$ via $z=x+i y \longleftrightarrow\left(\begin{array}{l}x \\ y\end{array}\right)$. We have

$$
f_{\mu}(z)=\lambda(\mu) z+R(z, \bar{z}, \mu)
$$

where $R(z, \bar{z}, \mu)$ consists of terms of order $\geq 2$ in $z, \bar{z}$. The following lemma is proved in [6].

LEMMA. Suppose that : (i) $f_{\mu}$ is six times continuously differentiable in all variables; (ii) (H1) and (H2) are satisfied; (iii) $\lambda_{0}^{n} \neq 1$ for $n=1,2,3,4,5$. Then we can find a new $z$-coordinate in which $f_{\mu}$ has the form

$$
f_{\mu}(z)=\lambda(\mu) z+\alpha(\mu) z^{2} \bar{z}+R_{5}(z, \bar{z}, \mu)
$$

where $R_{5}$ contains only terms of order $\geq 5$ in $z$ and $\bar{z}$.

Armed with this lemma, we look for an invariant curve for $f_{\mu}$. We try a circle as a first approximation:

$$
\left|f_{\mu}(z)\right|=|z|=r
$$


Let $\lambda(\mu)=\lambda_{0}\left(1+\mu \lambda_{1}+\ldots\right)$ where we assume for simplicity that $\lambda_{1}>0$. If $\lambda_{0}^{n} \neq 1$ for $1 \leq n \leq 5$, then by the lemma

$$
\left|f_{\mu}(z)\right| \simeq\left(1+\mu \lambda_{1}\right) r+\alpha(0) r^{3},
$$

so consider

$$
\left(1+\mu \lambda_{1}\right) r+\alpha(0) r^{3}=r \Longrightarrow r^{2}=-\mu \lambda_{1} / \alpha(0) \text {. }
$$

If $\alpha(0)<0$ one expects (and gets) a supercritical Hopf bifurcation: there is a closed curve, diffeormorphic to a circle, with radius the order of $\sqrt{\mu}$, which is invariant under $f_{\mu}$ for small $\mu>0$. Of course this closed curve is not a periodic orbit. Note that, if $\alpha(0)<0$, then the origin is an attractor when $\mu=0$, though the rate of attraction is not exponential. For this reason one speaks of a "weak attractor".

On the other hand, if $\alpha(0)>0$, then one expects (and gets) a subcritical Hopf bifurcation. This completes our brief review of the theory of Hopf bifurcation of maps.

We finish our discussion of bifurcation theory by considering a situation which, at first glance, looks more complicated than those considered above. Namely, suppose we have a one-parameter family of differential equations

$$
x^{\prime}=f_{\mu}(x) \quad\left(x \in \mathbf{R}^{k}, \quad \mu \in \mathbf{R}\right) .
$$

Suppose that $\left\{x_{\mu}(t)\right\}$ is a family of periodic solutions: thus $x_{\mu}(t+T)=x_{\mu}(t)$ where the (minimal) period $T$ may depend on $\mu$. Suppose that $x_{\mu}(t)$ is asymptotically stable for $\mu<0$ but not for $\mu>0$. Is there a new stable invariant set for $\mu>0$ ?

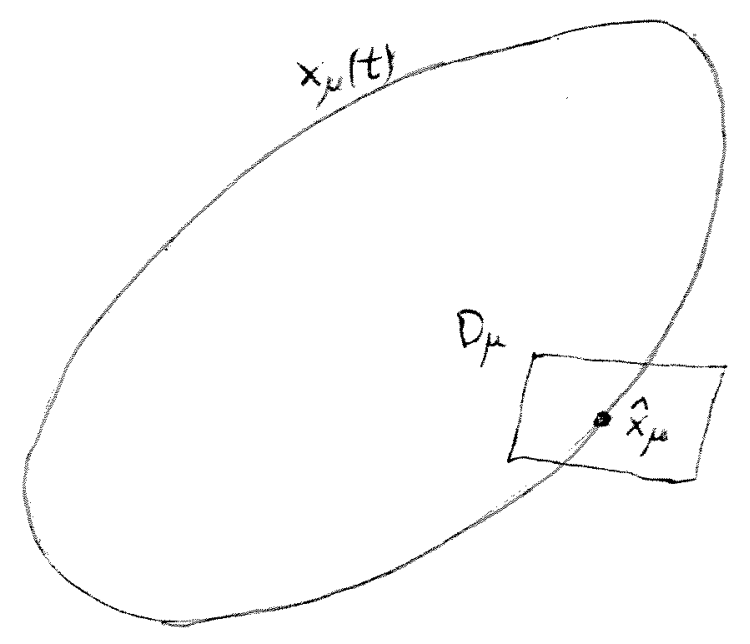

Figure 5 
We can reduce this problem to a bifurcation problem of mappings by the following device. Consider a family $D_{\mu}$ of small transversal sections to the periodic orbits $x_{\mu}(t)$ (Fig. 5). Let $\hat{x}_{\mu}$ be the (unique) point of intersection of $D_{\mu}$ with $x_{\mu}(t)$. If one solves $x^{\prime}=f_{\mu}(x)$ with initial condition in $D_{\mu}$, and if $D_{\mu}$ is small enough, then the solution must return to $D_{\mu}$. Moreover, $\hat{x}_{\mu}$ is mapped into itself under this "first return map", or Poincaré map. One considers the family $\left\{g_{\mu}\right\}$ of Poincaré maps: since $g_{\mu}\left(\hat{x}_{\mu}\right)=\hat{x}_{\mu}$, and since $\hat{x}_{\mu}$ is a stable fixed point of $g_{\mu}$ exactly when $x_{\mu}(t)$ is a stable periodic solution of $x^{\prime}=f_{\mu}(x)$, we have in fact reduced the original problem to a bifurcation problem of mappings.

It is interesting to note that, if the family $\left\{g_{\mu}\right\}$ of Poincaré maps satisfies the conditions for a supercritical Hopf bifurcation, then for $\mu>0$ there is a family of invariant stable 2-tori for the differential equations $x^{\prime}=f_{\mu}(x)$. (Recall that a 2-torus is the topological product of two circles.)

Now, clearly we can continue to pose our basic question when a family of invariant 2-tori loses stability. Indeed: is there a new stable invariant set? if so what are its properties?

Here there is no general theory available to answer the question. One thing that can happen is the appearance of hyperbolic invariant sets, with associated chaotic dynamics.

In the rest of this report, we discuss some aspects of the theory of hyperbolic invariant sets, then explain why they play a role in bifurcation problems.

III. Hyperbolic Systems. We begin the discussion by considering a special dynamical system, the 2-shift. This exhibits well-defined chaotic properties, and moreover is frequently found embedded in other dynamical systems.

Let $\Sigma$ be the set of bi-infinite sequences of zeroes and ones. That is, $\Sigma=\left\{\left(s_{i}\right)_{i=-\infty}^{\infty} \mid s_{i} \in\right.$ $\{0,1\}$ for each $i,-\infty<i<\infty\}$. Define the (lefi)-shift $T: \Sigma \rightarrow \Sigma$ as follows:

$$
T\left(\left(s_{i}\right)_{i=-\infty}^{\infty}\right)=\left(s_{i+1}\right)_{i=-\infty}^{\infty}
$$

Thus $T$ "picks up" the sequence and moves it one place to the left.

Now, the map $T$ is random in a quite elementary sense. Namely, suppose we know the digits of a sequence $\left(\bar{s}_{i}\right)_{i=-\infty}^{\infty}$ for, say, $|i| \leq i_{0}$. Suppose we apply $T$ to the sequence $j$ times where $j>i_{0}$. Then we have no knowledge of, say, $\left(T^{j} \bar{s}\right)_{i=0}$; that is, of the $0^{t h}$ digit of the $j$-times translated sequence $T^{j} \bar{s}$. Thus, if we think of $s_{0}$ as an initial "state", and of application of $T$ as corresponding to one unit of "time", then we see that after $j$ units of time all knowledge of the initial state is lost.

A quite large class of discrete dynamical systems contain 2-shifts. For example, any system with a transversal homoclinic point contains a 2 -shift.

We explain what a transversal homoclinic point is, and how shifts arise in their presence. The ideas and constructions are mainly due to Poincaré, Birkhoff, and Smale. 
Let $f: \mathbf{R}^{2} \rightarrow \mathbf{R}^{2}$ be a diffeomorphism with $f(0,0)=(0,0)$. Suppose that $(0,0)$ is a hyperbolic fixed point. That is, the matrix of first derivatives $D_{(0,0)} f$ is similar to a diagonal matrix:

$$
D_{(0,0)} f \simeq\left(\begin{array}{cc}
\lambda_{1} & 0 \\
0 & \lambda_{2}
\end{array}\right)
$$

and the eigenvalues $\lambda_{1}, \lambda_{2}$ satisfy $0<\left|\lambda_{2}\right|<1$ and $\left|\lambda_{1}\right|>1$. It is known that there exist stable and unstable manifolds $W^{s}, W^{u}$ passing through $(0,0)\left(\right.$ Fig. 6). Here $W^{s}=$ $\left\{q \mid f^{n}(q) \rightarrow(0,0)\right.$ as $\left.n \rightarrow+\infty\right\}$ and $W^{u}=\left\{q \mid f^{n}(q) \rightarrow(0,0)\right.$ as $\left.n \rightarrow \infty\right\}$. A transversal homoclinic point $p$ is a point of transversal intersection of $W^{s}$ and $W^{u}$.

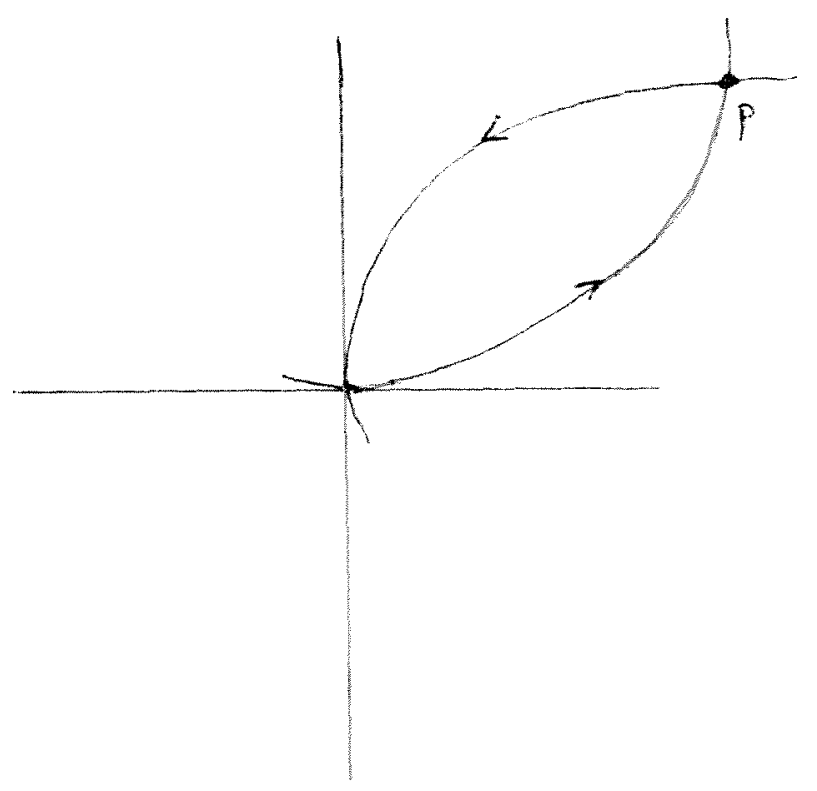

Figure 6

It is a fact, proved by Smale, that there is a (curvilinear) rectangle $R$ near $p$ and a positive integer $j$ such that $\left.f^{j}\right|_{R}$ is a horseshoe map. To explain this concept, consider a mapping $g$ of the plane to itself which stretches and bends a rectangle $R$ as in Fig. 7 . Define $H_{0} \cup H_{1}$. to be $f(R) \cap R$, and let $V_{0}=f^{-1}\left(H_{0}\right), V_{1}=f^{-1}\left(H_{1}\right)$. The $H_{i}$ s resp. $V_{i} \mathrm{~s}$ are called horizontal resp. vertical strips.

We will not explain in all detail how an invariant shift for $f$ is constructed, but we indicate the argument. Consider

$$
\widetilde{\Sigma}=\left\{x \in R \mid f^{i}(x) \in H_{0} \cup H_{1} \text { for all } i,-\infty<i<\infty\right\}
$$



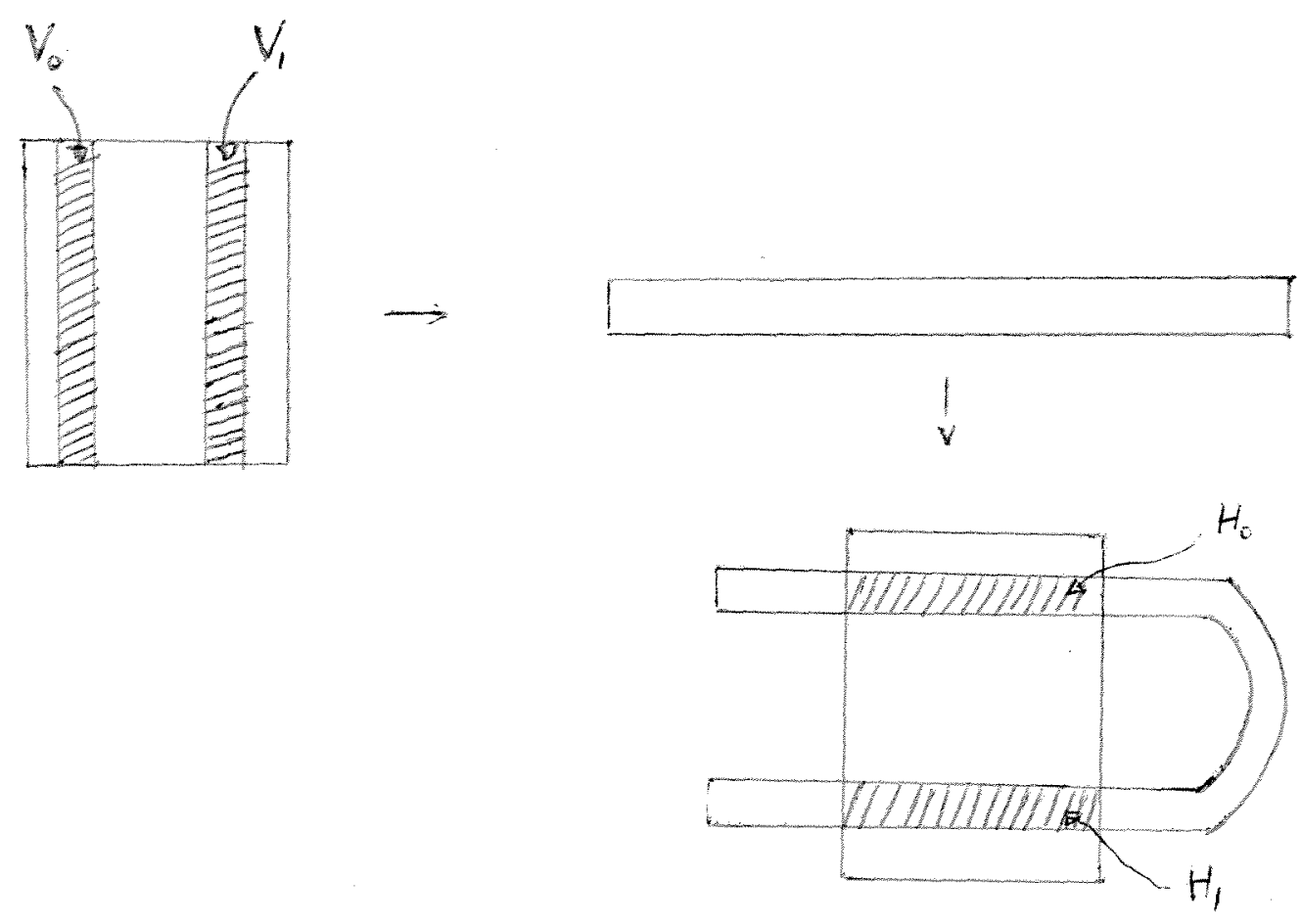

FIGURE 7

Then $\widetilde{\Sigma}$ is a compact invariant subset of $R$. It turns out that, if one considers positive iterates $i$, then one obtains that

$$
\bigcap_{i=0}^{\infty}\left\{x \mid f^{i}(x) \in H_{0} \cup H_{1}\right\}
$$

is a "Cantor set of vertical lines" obtained by throwing out vertical regions in a manner analogous to the way one eliminates middle thirds in the standard Cantor construction. Similarly

$$
\bigcap_{i<0}\left\{x \mid f^{i}(x) \in H_{0} \cup H_{1}\right\}
$$

is a "Cantor set of horizontal lines".

Now, $\widetilde{\Sigma}$ is by definition the set of all points of intersection of horizontal and vertical lines. The map $f$ restricted to $\widetilde{\Sigma}$ can be shown to be (isomorphic to) the standard 2-shift $(\Sigma, T)$, as follows. Define

$$
a: \widetilde{\Sigma} \rightarrow \Sigma: a(x)=\left(S_{i}\right)_{i=-\infty}^{\infty} \text { where } f^{i}(x) \in H_{S_{i}} .
$$

One can check that $a(f(x))=T(a(x))$ for all $x \in \tilde{\Sigma}$. One can also show that $a$ is bijective, and is in fact a homeomorphism with respect to a natural metric structure on $\Sigma$. Thus $a$ does indeed define an isomorphism between $\left.g\right|_{\widetilde{\Sigma}}$ and $(\Sigma, T)$. 


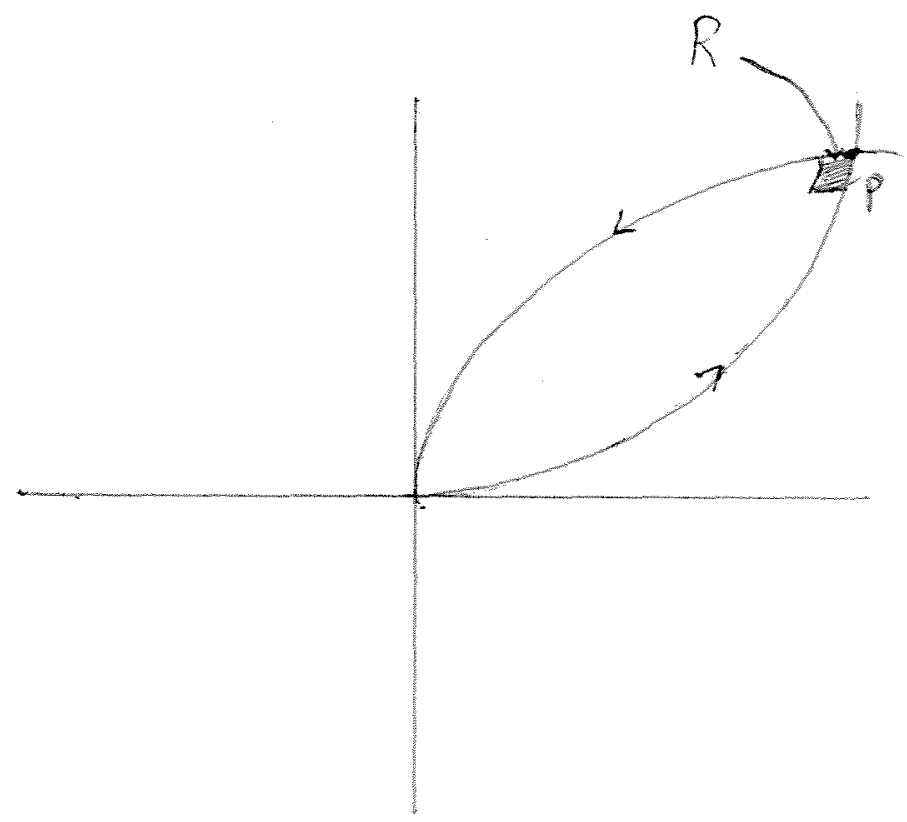

Figure 8

One can show that, if $p$ is a transversal homoclinic point, and if $R$ is a small rectangle as indicated in Fig. 8, then for large enough $j$, the map $g=\left.f^{j}\right|_{R}$ is a horseshoe map.

Let us consider a very simple example where transversal homoclinic points abound. Let $T^{2}=\mathbf{R}^{2} / \mathbf{Z}^{2}$ be the 2 -torus, and define

$$
f: T^{2} \rightarrow T^{2}: f\left(\begin{array}{l}
x_{1} \\
x_{2}
\end{array}\right)=\left(\begin{array}{ll}
2 & 1 \\
1 & 1
\end{array}\right)\left(\begin{array}{l}
x_{1} \\
x_{2}
\end{array}\right) .
$$

Since the determinant of the matrix $\left(\begin{array}{ll}2 & 1 \\ 1 & 1\end{array}\right)$ equals one, this formula does indeed define a mapping of the torus to itself which is a diffeomorphism. Note that the origin $x_{1}=0, x_{2}=0$ is a fixed point for $f$. It is a hyperbolic point, because the eigenvalues of $\left(\begin{array}{ll}2 & 1 \\ 1 & 1\end{array}\right)$ are $\lambda_{1}=1 / 2(3+\sqrt{5}), \lambda_{2}=1 / 2(3-\sqrt{5})$. The stable resp. unstable manifolds are

$$
\begin{aligned}
& W^{s}=\{(-2 t,(\sqrt{5}+1) t) \mid t \in \mathbf{R}\} \subset T^{2}, \\
& W^{u}=\{(2 t,(\sqrt{5}-1) t) \mid t \in \mathbf{R}\} \subset T^{2} .
\end{aligned}
$$

These manifolds (curves) wind densely on the torus; moreover they intersect transversely in infinitely many points which form a dense subset of the torus. There is an inrariant shift in every neighborhood of each such transversal homoclinic point. 
The example we have discussed is the standard Anosov diffeomorphism.

Let us now define and briefly discuss the theory of (uniformly) hyperbolic invariant sets. These are quite well understood, occur fairly often, and have a structure which most anyone would describe as chaotic.

Let $f: \mathbf{R}^{k} \rightarrow \mathbf{R}^{k}$ be a diffeomorphism. Let $\wedge \subset \mathbf{R}^{k}$ be a compact invariant set which is invariant under $f: f(\wedge)=\wedge$. We say that $\wedge$ is hyperbolic if the tangent bundle $T(\wedge)=\wedge \times \mathbf{R}^{n}$ admits a splitting

$$
T(\wedge)=E^{s} \oplus E^{u}
$$

where: (i) $D f\left(E^{s}\right)=E^{s}, D f\left(E^{u}\right)=E^{u}$ (here $D f$ denotes the derivative map of $f$ ); (ii) $\left\|\left.D f^{n}\right|_{E^{\prime}}\right\| \leq K \exp (-\beta n)$ and $\left\|\left.D f^{-n}\right|_{E^{u}}\right\| \leq K \exp (-\beta n)$ for all $n \geq 1$ where $K^{\prime}, \beta$ are positive constants.

The basic theorem which allows the detailed study of hyperbolic invariant sets is the

Stable Manifold Theorem. Let $\wedge$ be hyperbolic and let $x \in \wedge$. There are smooth manifolds $W^{s}(x)$ resp. $W^{u}(x)$ which are tangent at $x$ to $E^{s}(x)$ resp. $E^{u}(x)$ with the following properties. First, they are invariant: $f\left(W^{s}(x)\right) \subset W^{s}(f(x))$ and $f\left(W^{v}(x)\right) \subset$ $W^{u}(f(x))$. Second, $W^{s}(x)$ consists exactly of those points $y \in \mathbf{R}^{k}$ such that $\| f^{n}(y)-$ $f^{n}(x) \| \rightarrow 0$ as $n \rightarrow \infty$, while $W^{u}(x)$ consists exactly of those points $y \in \mathbf{R}^{k}$ such that ||$\left.f^{n}(y)-f^{n}(x)\right) \mid \rightarrow 0$ as $n \rightarrow-\infty$.

With the stable manifold theorem at hand, we can describe further developments of the theory. Suppose that $\wedge$ is hyperbolic, "indecomposable", and maximal with respect to this latter property. See Guckenheimer and Holmes [5] for a discussion of indecomposability.

Definition. A rectangle is a closed subset $R$ of $\wedge$ such that, if $x, y \in R$, then

$$
W_{\epsilon}^{s}(x) \cap W_{\epsilon}^{u}(y)=\{p\}, \text { a singleton, and moreover } p \in R
$$

Here the subscript $\epsilon$ refers to local stable and unstable manifolds: $y \in W_{\epsilon}^{s}(x)$ if and only if $y \in W^{s}(x)$ and $\left\|f^{n}(x)-f^{n}(y)\right\| \leq \epsilon$ for all $n \geq 0$, and analogously for $W_{\epsilon}^{u}(x)$. 


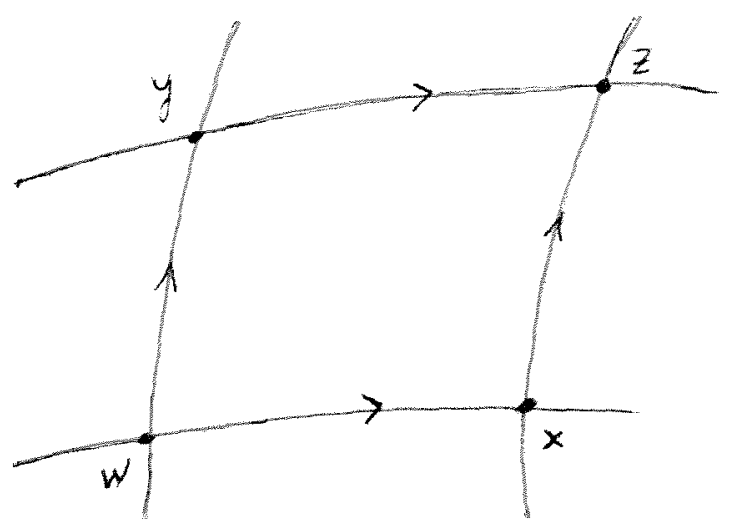

Figure 9

We can construct rectangles as follows (Fig. 9). Let $x \in \wedge$. There exist $\epsilon>0 . \delta>0$ such that, if $R=\left\{y \mid\right.$ there exist $w \in W_{\delta}^{s}(x), z \in W_{\delta}^{u}(x)$ with $\left.\{y\}=W_{\epsilon}^{u}(w) \cap W_{\epsilon}^{z}(z)\right\}$, then $R$ is a rectangle. Once again see Guckenheimer and Holmes [5] for a detailed discussion.

We need still another basic definition.

DEFINITION. A Markov partition for $\wedge$ is a collection $\left\{R_{1}, \ldots, R_{m}\right\}$ of rectangles such that:

(i) $\wedge=\bigcup_{i=1}^{m} R_{i}$;

(ii) Int $R_{i} \cap$ Int $R_{j}=\phi$ if $i \neq j$;

(iii) $f\left(W^{u}\left(x, R_{i}\right)\right) \supset W^{u}\left(f(x), R_{j}\right)$ and $f\left(W^{s}\left(x, R_{i}\right)\right) \subset W^{s}\left(f(x), R_{j}\right)$ if $x \in$ Int $R_{i}$ and $f(x) \in \operatorname{Int} R_{j}$.

One now follows Bowen [1], who proved the basic result that a maximal indecomposable hyperbolic invariant set admits a Markov partition. (In the horseshoe example, a Markov partition for $\widetilde{\Sigma}$ is given by $\left\{H_{0}, H_{1}\right\}$.)

If $\wedge$ has a Markov partition $\left\{R_{1}, \ldots, R_{m}\right\}$, define an $m \times m$-matrix $A$ as follows:

$$
\begin{array}{ll}
A_{i j}=0 & \text { if Int } R_{i} \cap f^{-1} \text { Int } R_{j}=\emptyset \\
A_{i j}=1 & \text { if Int } R_{i} \cap f^{-1} \text { Int } R_{j} \neq \emptyset
\end{array}
$$


Next define the subshift of finite type $\Sigma_{A}$ as follows. The space $\Sigma_{A}$ is the set of sequences $\left(s_{i}\right)_{i=-\infty}^{\infty}$ such that $s_{i} \in\{1,2, \ldots, m\}$ for all $i$, and such that $A_{s_{i} s_{i+1}}=1$ for all $i$. Thus the matrix $A$ is used to pick out a subset of the full shift on $m$ symbols (and not just on 2 symbols as considered earlier). Define $T: \Sigma_{A} \rightarrow \Sigma_{A}$ just as in the case of the 2-shift, namely $T\left(\left(s_{i}\right)_{i=-\infty}^{\infty}\right)=\left(s_{i+1}\right)_{i=-\infty}^{\infty}$.

Now, one defines a mapping $\pi: \Sigma_{A} \rightarrow \wedge$ as follows:

$$
\pi\left(\left(s_{i}\right)_{i=-\infty}^{\infty}\right)=\bigcap_{i=-\infty}^{\infty} f^{-1}\left(\boldsymbol{R}_{s_{i}}\right) .
$$

It turns out that the intersection on the right consists of exactly one point, so $\pi$ is indeed a well-defined mapping. Bowen proved that $\pi$ is surjective, and moreover is injective when restricted to the inverse image

$$
\pi^{-1}\left(\bigcap_{i=-\infty}^{\infty} f^{i}\left(\bigcup_{j=1}^{m} \operatorname{Int} R_{j}\right)\right) \subset \Sigma_{A} .
$$

(The Baire category theorem shows that the set on the left is residual in $\Sigma_{A}$ ). Furthermore $\pi \circ T=f \circ \pi$. Thus $\left.f\right|_{\wedge}$ reflects properties of the highly chaotic flow $\left(\Sigma_{A}, T\right)$.

This completes our review of the theory of invariant sets with a uniform hyperbolic structure. There is also a theory of invariant sets with a hyperbolic structure which is not uniform but only "measurable". This theory is based upon fundamental results of Oseledec [9] and Pesin [10]. We will not discuss this theory here.

IV. Hyperbolicity in Bifurcation Problems. Now we return to bifurcation theory, and indicate in a general way why hyperbolic behavior is to be expected in situations where a 2-torus loses stability in a 1-parameter family of differential equations, or an invariant curve loses stability in a 1-parameter family of difference equations. We take the classical (though perhaps not vogueish) approach of linearizing the non-linear system around the invariant torus or curve, studying the linearized equations, then using linear information together with certain assumptions about the non-linearity to gain information about the nature of stable solutions and transfer of stability. In what follows we discuss differential equations, assuring the reader that completely analogous considerations are valid for difference equations.

We study a situation analogous to that occuring in the Hopf bifurcation. Consider once again a one-parameter family of differential equations

$$
x^{\prime}=f_{\mu}(x) \quad\left(x \in \mathbf{R}^{k}, \mu \in \mathbf{R}\right) .
$$

We may as well assume that $k$ is "large", i.e., at least 4 . Suppose that there is a family $x=v_{\mu}(t)$ of solutions such that $Y_{\boldsymbol{\mu}}=\operatorname{cls}\left\{v_{\mu}(t) \mid t \in \mathbf{R}\right\}$ is homeomorphic to a 2-torus for each $\mu \in \mathbf{R}$. We consider the linearized equations

$$
u^{\prime}=D f_{\mu}\left(v_{\mu}(t)\right) u
$$


Assume that $Y_{\mu}$ is asymptotically stable for $\mu<0$ but is unstable for $\mu>0$. Assume further that "stability is lost in exactly two directions". Though the precise formulation of this condition is not as transparent as it is in the case of the Hopf bifurcation, we take it to mean that equation (2) can be transformed into the form

$$
\left(\begin{array}{l}
x \\
z
\end{array}\right)^{\prime}=\left(\begin{array}{cc}
B_{\mu}\left(v_{\mu}(t)\right) & 0 \\
* & Z_{\mu}\left(v_{\mu}(t)\right)
\end{array}\right)\left(\begin{array}{l}
x \\
z
\end{array}\right)
$$

where $x \in \mathbf{R}^{2}, z \in \mathbf{R}^{k-2}$, and the $z$-directions are "unimportant" for the stability analysis. In particular the directions tangent to $Y_{\lambda}$ are included in the $z$-variables.

We remark that Sell [12] has considered conditions on $Y_{\mu}$ which ensure that such a reduction to triangular form can be made.

The above discussion can be summarized by saying that we assume that the stability analysis can be reduced to the study of a two-dimensional linear problem

$$
x^{\prime}=B_{\mu}\left(v_{\mu}(t)\right) x \quad x \in \mathbf{R}^{2}
$$

To study this problem, let $\tau_{\mu}$ denote the flow on $Y_{\mu}$ induced by the vector field $f_{\mu}$. Thus $t \rightarrow v_{\mu}(t)$ defines one orbit of this flow. We are going to assume (though the assumption can be weakened considerably) that the flow on $Y_{\mu}$ is quasi-periodic. This means, after a continuous change of coordinates, the flow $\tau_{\mu}$ has the following form:

$$
\tau_{\mu}\left(\left(\psi_{1}, \psi_{2}\right), t\right)=\left(\psi_{1}+t, \quad \psi_{2}+\gamma(\mu) t\right)
$$

Here $0 \leq \psi_{1} \leq 2 \pi, \quad 0 \leq \psi_{2} \leq 2 \pi$ are $2 \pi$-periodic coordinates on the two-torus $Y_{\mu}$, and $\gamma(\mu)$ is a real number called a frequency.

We need a definition: for fixed $\mu$, equation $(3)_{\mu}$ has an exponential dichotomy if there is a quasi-periodic change of variables $x=P(t) y\left(y \in \mathbf{R}^{2}, P(t)\right.$ a non-singular $2 \times 2$ matrix $)$ such that, in the $y$-coordinates, $(3)_{\mu}$ becomes

$$
y^{\prime}=\left(\begin{array}{ll}
a & 0 \\
0 & b
\end{array}\right) y
$$

with constants $a \neq b$. Let $\theta$ be the polar angle in the $x$-plane. A moment's thought shows that, if $(3)_{\mu}$ has an exponential dichotomy, then there are exponentially attracting and repelling solutions $\theta_{+}(t), \theta_{-}(t)$ of the differential equation for $\theta$ defined by equation (3) (if $(3)_{\mu}$ already has the form (4), and if $a>b$, then $\theta_{+}(t) \equiv 0$ and $\theta_{-}(t) \equiv \pi / 2$.)

Now, consider a fixed reference 2-torus $Y$ to which all the $Y_{\mu}$ are homeomorphic. Let $\left(\psi_{1}, \psi_{2}\right)$ be angular coordinates on $Y$. The differential equations $(3)_{\mu}$ define a curve $c$ in the space $\mathcal{A}=\mathbf{R} \times \mathcal{C}_{2}$, where $\mathbf{R}$ is the space of frequency vectors $\gamma$ and $\mathrm{C}_{2}$ is the space of $2 \times 2$ matrix-valued functions $B$ defined on $Y$. (One can show that, for each $\mu$, the 
function $B_{\mu}\left(v_{\mu}(t)\right)$ defined along the orbit $t \rightarrow v_{\mu}(t)$ actually extends to a continuous function $B_{\mu}\left(\psi_{1}, \psi_{2}\right)$ defined on all of $Y$.) Thus $c(\mu)=\left(\gamma(\mu), B_{\mu}(\cdot)\right) \in \mathcal{A}$.

We can now state a key result [7]: for a "generic" curve $c=c(\mu)$ in $\mathcal{A}$, there is an open and dense set $E=\{\mu\}$ of parameter values $\mu$ for which equation (3) $\mu$ has an exponential dichotomy. Thus a "generic" bifurcation problem $(2)_{\mu}$ gives rise to linearized systems $(3)_{\mu}$ which have exponential dichotomy for an open dense set of $\mu$.

The intuitive reason that this result has implications for bifurcation problems is the exponential attraction resp. repulsion of $\theta_{+}(t)$ resp. $\theta_{-}(t)$. Because of this exponential attraction/repulsion, one expects that, near $Y_{\mu}$, the angular evolution of solutions of the non-linear equation (2) $\mu$ will resemble the angular evolution of solutions of the linearized equation $(3)_{\mu}$. And indeed, examples due to Ying-Fei Yi show that this intuition concerning the time evolution of $\theta$ can be used to produce examples where, for an open dense set of $\mu>0$, equation $(2)_{\mu}$ has transversal homoclinic orbits (with corresponding shifts) in each neighborhood of $Y_{\mu}$. In general one can expect to find markedly hyperbolic behavior in the vicinity of $Y_{\mu}$ for $\mu>0$.

\section{BIBLIOGRAPHY}

[1] Bowen, R., Markov partitions for Axiom A diffeomorphisms, Am. J. Math. 92 (1970), $725-747$.

[2] Collet, P., Eckmann, J.P., Iterated Maps on the Interval as Dynamical Systems, Birkhäuser, Boston (1980).

[3] Feigenbaum, M., Quantitative universality for a class of non-linear transformations, J. Stat. Phys. $19(1978), 25-52$.

[4] Golvbrtsky, M., Sch Aefer, D., Singularities and Groups in Bifurcation Theory, Vol. I. Appl. Math. Sci. 51, Springer-Verlag, New York (1985).

[5] Guckenhemer, J., Holmes, P.,, Nonlinear Oscillations, Dynamical Systems, and Bifurcations of Vector Fields, Springer-Verlag, New York (1983).

[6] Iooss, G, Bifurcations of Maps and Applications, North-Holland, New York (1979).

[7] Johnson, R., Hopf bifurcation from non-periodic solutions of differential systems I: linear theory, Dyzamics and Diff. Eqns. 1 (1989), 179-198.

[8] Marsden, J., Mc Cracken, M., The Hopf Bifurcation and its Applications, Appl. Math. Sci. 19, Springer-Verlag, New York (1976).

[9] Osetedec, V., A multiplicative ergodic theorem. Characteristic Lyapounov exponents of dynamical systems, Proc. Moscow Math. Soc. 19 (1968), 179-210.

[10] PEsIN, JA., Characteristic Lyapunov exponents and smooth ergodic theory, Russ. Math. Surveys 32 (1977), 55-114.

[11] SACKER, R., On invariant surfaces and bifurcation of periodic solutions of ordinary differential equations, New York Univ. Technical Report IMM-NYU (1964).

[12] SeIl, G., Bifurcation of higher-dimensional tori, Archive Rat. Mech. Anal. 69 (1979), $199-230$. 